\title{
Spatio-temporal correlation Super-Resolution Optical Fluctuation Imaging
}

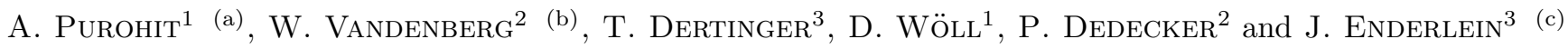 \\ 1 Institute for Physical Chemistry, RWTH Aachen University, Landoltweg 2, 52074 Aachen, Germany. \\ 2 Department of Chemistry, KU Leuven, Celestijnenlaan 200G, 3001 Heverlee, Belgium. \\ 3 III. Institute of Physics - Biophysics, Georg August University, 3707r Göttingen, Germany.
}

PACS $07.60 . \mathrm{Pb}$ - Conventional optical microscopes

PACS $42.30 . \mathrm{Va}$ - Image forming and processing

PACS 87.64.M- - Optical microscopy

\begin{abstract}
We describe a new concept of multimodal super-resolution imaging which combines the cumulant analysis from Super-resolution Optical Fluctuation Imaging (SOFI) with the imprinting of three-dimensional, spectral or other information into peculiar Point-Spread Function patterns. This concept allows for encoding multidimensional or multimodal information into a single image plane and to extract this information by an appropriate spatio-temporal correlation analysis of emitter fluctuations. Here, we develop the general theory of this concept, and present proof-ofprinciple experiments of three-dimensional super-resolution imaging.
\end{abstract}

Introduction. - The field of super-resolution optical microscopy has seen a revolution over the past twenty years, first with the invention of STimulated Emission Depletion (STED) microscopy [1,2], and later with the development of Single-Molecule Localization Microscopy (SMLM), such as (fluorescence) Photo-Activatable Localization Microscopy ((f)PALM) [3,4], STochastic Optical Reconstruction Microscopy (STORM) [5], and direct STORM (dSTORM) [6]. One recent method which also uses the optical switching of emitter intensity but without single-molecule localization is Super-resolution (or Stochastic) Optical Fluctuation Imaging (SOFI) [7], which uses the statistical independence of the stochastic blinking of emitters for increasing spatial resolution via a temporal cumulant analysis.

All these methods aim, at first order, to improve the lateral resolution of a microscope. Gaining structural information and increasing resolution along the optical axis requires additional efforts. In STED microscopy, threedimensional images are obtained by repeatedly scanning a sample with shifted focal planes, but enhancing also the resolution along the optical axis requires appropriate phase-plates that squeeze the STED detection volume

\footnotetext{
(a) Equal contribution first author

(b) Equal contribution first author

(c) E-mail: jenderl@gwdg.de
}

along the optical axis, see e.g. [8]. Thus, for achieving both increased resolution laterally as well as axially, a STED microscope needs a sophisticated combination of both laterally as well as axially squeezing STED-focus shaping [9]. SOFI automatically increases the optical resolution along all three dimensions (already second-order SOFI introduces optical sectioning capability into a conventional wide-field microscope), but requires also a shifting of the focal plane for recording a three-dimensional image $[10,11]$. In SMLM, three-dimensional information is gained by methods that can localize a single emitter not only laterally, but also along the optical axis. Among the most popular approaches are astigmatic imaging [12], biplane imaging [13], and helical [14] or more general wavefront shaping [15].

Especially the latter is of particular interest for the current paper: In helical wavefront shaping, one inserts a special phase-mask into the detection path of a wide field microscope, which modulates the wavefront of detected light in such a way that a molecule's image (its Point Spread Function or PSF) shows a peculiar pattern in the image plane which encodes the axial position of the emitter. A fit of the center position of this pattern then yields the lateral position of the molecule, whereas fitting the pattern's shape yields its axial position.

In the current paper we propose a new concept of threedimensional super-resolution imaging which combines the 


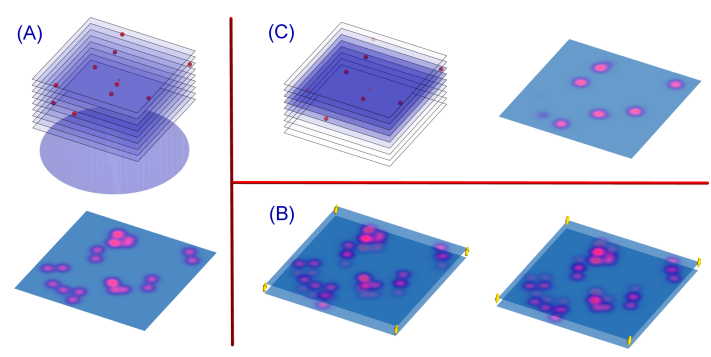

Fig. 1: Schematic of stSOFI: (A) A three-dimensional sample with stochastically blinking emitters is imaged onto a camera by using a specialized phase plate that encodes the axial position of an emitter into a peculiar shape of its PSF (shown here: a double spot pattern where the orientation angle of the pattern in the image plane correlates with the axial position of the emitter). (B) A recorded movie is analyzed by applying a series of spatio-temporal correlation analysis, where a given shift and orientation of the spatial correlation selects a small axial range along the optical axis, see $(\mathrm{C})$. cumulant analysis of SOFI with imprinting the axial position of an emitter into its PSF. For extracting the axial position information, spatio-temporal correlation SOFI (stSOFI) applies a spatio-temporal cumulant analysis to a recorded single-plane movie $[16,17]$ (very similar to what is done in spatio-temporal image correlation spectroscopy or STICS [18]), which then results in a three-dimensional image of the sample without any need of focal-plane scanning, see Fig.1. This general idea can also be applied to other image information content: For example, by encoding the spectral emission of an emitter into its PSF shape, one can use a similar stSOFI approach for generating multi-spectral super-resolution images, without the need of recording several spectrally resolved images.

In the next paragraph, we explain the theoretical concept of multimodal stSOFI on the example of threedimensional imaging with helical wavefront shaping, and after that present proof-of-principle experiments for threedimensional stSOFI.

Theory. - Let us first briefly recall the concept of conventional SOFI. In wide-field microscopy, the image $I(\boldsymbol{\rho}, t)$ on the detector as a function of pixel position $\boldsymbol{\rho}$ and time of acquisition $t$ is the sum of the PSFs of all emitters in a sample,

$$
I(\boldsymbol{\rho}, t)=\sum_{\text {all emitters }} U\left(\boldsymbol{\rho}-\boldsymbol{\rho}_{j}^{\prime}, z_{j}^{\prime}\right) s_{j}(t)
$$

where $U(\boldsymbol{\rho}, z)$ is the image (PSF) of an emitter located on the optical axis at position $z$ along it, $\boldsymbol{\rho}_{j}^{\prime}$ and $z_{j}^{\prime}$ are the lateral and axial position of the $j^{\text {th }}$ emitter, and $s_{j}(t)$ is its brightness at time $t$. Here, it is also assumed that the focal plane is positioned at $z=0$ in the sample. In its simplest form, second-order SOFI, a super-resolved image is calculated from a recorded movie of images $I(\boldsymbol{\rho}, t)$ via

$$
I_{\mathrm{SOFI}}^{(2)}(\boldsymbol{\rho})=\langle\delta I(\boldsymbol{\rho}, t) \delta I(\boldsymbol{\rho}, t+\Delta t)\rangle_{t, \Delta t>0}
$$

where the $\delta I(\boldsymbol{\rho}, t)=I(\boldsymbol{\rho}, t)-\left\langle I\left(\boldsymbol{\rho}, t^{\prime}\right)\right\rangle_{t}^{\prime}$ is the temporal deviation from the mean, and the angular brackets denote time averaging over $t$ and summation of lag times $\Delta t$. If the emitters blink stochastically, but statistically independent form each other, with identical correlation function $\epsilon^{2}=\left\langle s_{j}(t) s_{j}(t+\Delta t)\right\rangle_{t, \Delta t>0}$, one obtains

$$
I_{\mathrm{SOFI}}^{(2)}(\boldsymbol{\rho})=\epsilon^{2} \sum_{\text {all emitters }} U^{2}\left(\boldsymbol{\rho}-\boldsymbol{\rho}_{j}^{\prime}, z_{j}^{\prime}\right)
$$

which demonstrates that already an image in second-order SOFI is formed with the square of the original PSF of the microscope (which translates, after appropriate deconvolution, into a ca. two-fold enhancement in resolution), while $\epsilon$ is proportional the mean brightness of the emitters. This concept can be extended to higher order correlation (cumulants), and several modifications have been published for dealing with effect of detector pixelation $[16,19]$ or brightness linearization (balanced SOFI, see Ref. [20]). Here, we will restrict our consideration to the simplest form of second-order SOFI as embodied by eqs. (2) and (3).

Let us next assume that imaging is done while using a helical wavefront shaper, which converts the image of a single emitter into a dumbbell-shaped PSF where the orientation of the dumbbell depends on the axial position $z$ of the emitter [21]. For the sake of simplicity, let us model this PSF by a superposition of two two-dimensional Gaussians as

$$
U(\boldsymbol{\rho}, z)=\sum_{\kappa= \pm 1} \exp \left(-|\boldsymbol{\rho}+\kappa \mathbf{v}(z)|^{2} / 2 \sigma^{2}\right)
$$

where the $z$-dependent shift vector $\mathbf{v}(z)$ is given by $\mathbf{v}(z)=$ $a\{\cos (\pi z / h), \sin (\pi z / h)\}$. Here, $h$ is the dynamic range of the helical wavefront shaper: When moving an emitter along the optical axis by a distance $h$, the PSF becomes the same again. In its application of helical-wavefront shaping to SMLM, one fits the orientation of the PSFdumbbell of a single emitter and thus obtains its axial position, modulo $h$. But one can also use this shape-encoded axial-position information in the context of SOFI. Let us calculate a modified stSOFI image by

$$
\begin{aligned}
& I_{\mathrm{stSOFI}}^{(2)}(\boldsymbol{\rho}, z)= \\
& =\sum_{\kappa= \pm 1}\langle I(\boldsymbol{\rho}+\kappa \mathbf{v}(z), t) I(\boldsymbol{\rho}-\kappa \mathbf{v}(z), t+\Delta t)\rangle_{t, \Delta t}
\end{aligned}
$$

where one performs the temporal correlation for pairs of pixels with a mutual shift $2 \mathbf{v}(z)$ to each other which corresponds to the distance between the two dumbbell maxima 


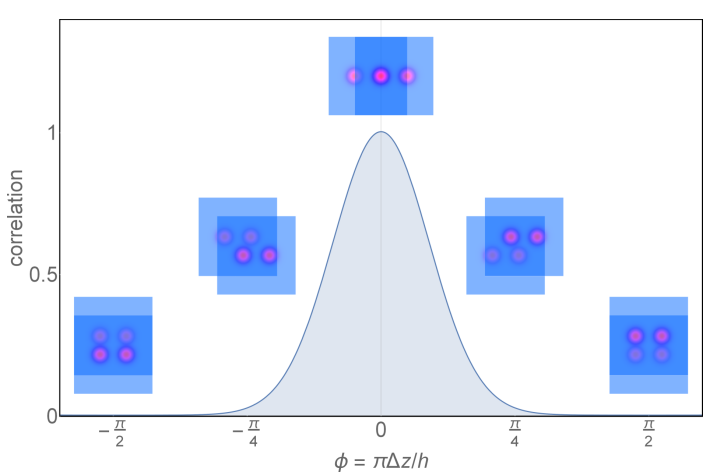

Fig. 2: Example of the overlap function, eq. (8), for $a=1$ and $\sigma=0.4$. Shown is the dependence of the function on angular variable $\phi=\pi \Delta z / h$. The insets visualize the different relative shifts of raw image pairs when performing the spatio-temporal correlation of stSOFI, for $\phi$-values, from left to right, of $-\pi / 2$, $-\pi / 4,0, \pi / 4$, and $\pi / 2$.

for an emitter at axial position $z$. This yields a superresolved image of the sample which is sensitive to the axial position of emitters. The farther away an emitter is from a plane at axial position $z$, the less its emission will contribute to the correlation in eq. (5). In quantitative terms, the contribution of an emitter at axial position $z^{\prime}$ to $I_{\mathrm{stSOFI}}^{(2)}(\boldsymbol{\rho}, z)$ will be proportional to the mutual overlap $C\left(z^{\prime}-z \mid a, \sigma, h\right)$ of two copies of its dumbbell PSF that are spatially shifted to each other along vector $\pm \mathbf{v}(z)$. Here, a vertical bar divides the main argument $z^{\prime}-z$ of the function from the parameters $a, \sigma$, and $h$. This overlap function can be calculated by

$$
\begin{aligned}
C & (\Delta z \mid a, \sigma, h)= \\
& =\iint d \boldsymbol{\rho} U[\boldsymbol{\rho}-\mathbf{v}(\Delta z), 0] U[\boldsymbol{\rho}+\mathbf{v}(\Delta z), 0]
\end{aligned}
$$

where we have introduced the abbreviation $\Delta z=z^{\prime}-z$. An example of this function is shown in Fig. 2.

Using the double-Gaussian approximation of the PSF from eq. (4), this overlap function can be computed analytically and reads

$$
\begin{gathered}
C(\Delta z \mid a, \sigma, h)=\exp \left[-\kappa^{2}(4 \cos \phi+\cos 2 \phi+5) / 2\right] \\
\times\left\{2 \exp \left(4 \kappa^{2} \cos ^{4} \phi / 2\right)+\exp \left(\kappa^{2} \cos ^{2} \phi\right)\right. \\
\left.+\exp \left[\kappa^{2}(8 \cos \phi+\cos 2 \phi+1) / 2\right]\right\}
\end{gathered}
$$

where we have used the abbreviations $\kappa=a / \sigma$ and $\phi=$ $\pi \Delta z / h$. This overlap function $C(\Delta z \mid a, \sigma, h)$ is periodic in $\Delta z$ with period $h$.

To quantify the optical sectioning capability of stSOFI, we compute the variance $\left\langle\Delta z^{2}\right\rangle$ using the overlap function $C(\Delta z \mid a, \sigma, h)-C(h / 2 \mid a, \sigma, h)$ as weight-function (where we have subtracted its offset at $\Delta z=h / 2$ ), and then estimate the full width at half maximum (FWHM) of the optical sectioning as $2 \sqrt{2 \ln 2\left\langle\Delta z^{2}\right\rangle}$, see Fig. 3. There, we can

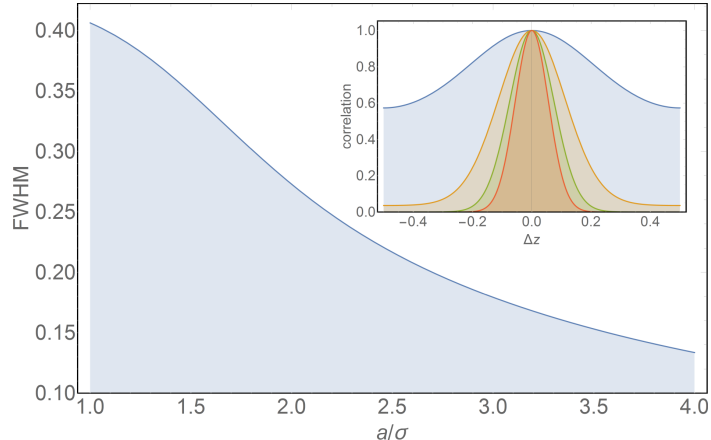

Fig. 3: Optical sectioning capability of stSOFI as a function of the ratio $a / \sigma$ and for $h=1$. The inset shows four different curves $C(\Delta z \mid a, \sigma, h)$ for values $a / \sigma$ of $1,2,3$ and 4 (the larger this value, the more narrow is the corresponding distribution of $C(\Delta z \mid a, \sigma, h))$. The large figure shows the full width at half maximum (FWHM) of these distributions as a function of $a / \sigma$. Because the whole dynamic range $h$ was set to the numerical value of one, the figure shows that for values of $a / \sigma$ smaller than $\sim 2.2$, the function $C(\Delta z \mid a, \sigma, h)$ has a FWHM value larger than one fourth of the dynamic range $h$ and does not sufficiently fall off to zero for $\Delta z= \pm h / 2$. In that case, optical sectioning will be imperfect.

seen that the z-sectioning capability breaks down as soon as the ratio of lobe distance to lobe diameter $a / \sigma$ becomes smaller than $\sim 0.22$, below which the FWHM of the curve $C(\Delta z \mid a, \sigma, h)$ becomes larger than $h / 4$, i.e. larger than one quarter of the dynamic range $h$ of the helical wavefront shaper. For relating the FWHM values given by Fig. 2 in units of $h$ to real length values, we have to estimate realistic values for $h$. In a conventional diffraction-limited microscope, the maximum spatial frequency along the optical axis that is still transmitted through an objective with numerical aperture N.A. $=n \sin \Theta$ is $k_{\max }=k(1-\cos \Theta)$. Here, $n$ is the refractive index of the imaging medium for which the objective is designed, $\Theta$ is the maximum half angle of light collection of the objective, and $k=2 \pi \lambda / n$ is the length of the wavevector for a vacuum wavelength $\lambda$. From this maximum transmitted spatial frequency, on can estimate the the minimum achievable value for $h$ will be

$$
h_{\text {min }}=\frac{\lambda}{n\{1-\cos [\arcsin (n / \text { N.A. })]\}}
$$

As an example, for an emission wavelength around $\lambda=$ $600 \mathrm{~nm}$ and a numerical aperture of N.A. $=1.2$ for a water immersion objective $(n=1.33)$, we thus arrive at a value of $h_{\min }$ around $0.8 \mu \mathrm{m}$. This values defines the maximum sectioning capability, but sets also a limit to the dynamic range over which one can uniquely resolve the axial distance. Actual helical wavefront shapers will use a value larger than this minimum, to extend the dynamic range to values close to the depth of focus of the objective. 
Experimental results. - For measurements of three-dimensional stSOFI, we used a wide-field epifluorescence microscope (IX83, Olympus). Fluorescence excitation is done with a $488 \mathrm{~nm}$ Laser (Cobalt Jive, 200 $\mathrm{mW}$ ), by focusing the laser light onto the back focal plane of the microscope's objective $(100 \times 1.3 \mathrm{NA}$ oil immersion objective UPLFLN-2 O, Olympus) using a plano-convex lens of focal length $500 \mathrm{~mm}$. A dichroic mirror (zt 488 $\mathrm{RDC}, \mathrm{AHF}$ ) reflects the excitation beam and transmits the fluorescence emission. The detection path consists of a Double Helix SPINDLE ${ }^{\mathrm{TM}}$ module (Double Helix Optics, Boulder, USA), a BP 617/73 Brightline HC band pass emission filter (AHF), and an EMCCD camera (Andor ixon Ultra 897). The SPINDLE module was placed between the exit of the microscope and the EMCCD camera using standard C-mounts. It consists of a $4 \mathrm{f}$ imaging system composed of two lenses of equal focal lengths. A fabricated phase mask specific to the emission wavelength is placed in the Fourier plane of the $4 \mathrm{f}$ system. The SPINDLE module converts the standard point spread function of a single emitter into two lobes (dumbbell pattern) [22]. The orientation angle of these two lobes depends on the axial position of the emitter, and for the used SPINDLE module, an axial shift of $3.18 \mu \mathrm{m}$ results in a rotation of $180^{\circ}$. Thus, the parameter $h$ from the Theory section is equal to $3.18 \mu \mathrm{m}$, and is thus roughly three times larger than the theoretically possible minimum. The mean size of one dumbbell lobe (as fitted with a symmetric Gaussian) is 1.4 pixel, corresponding to $0.224 \mu \mathrm{m}$ (for the used magnification, one camera pixel corresponds to $160 \mathrm{~nm}$ in sample space), and the average lobe distance is ca. 6.5 pixel or $1.04 \mu \mathrm{m}$, see also Fig.4. Thus, the parameter ratio $a / \sigma$ is $\sim 0.22$, close to the limit where complete optical sectioning should still be possible, see Fig. 3.

For sample preparation, we spin-coated a dilute solution of fluorescent quantum dots (QDs, Qdot 625 ITK Carboxyl Quantum Dots, Thermo Fisher Scientific) with diameters between $15 \mathrm{~nm}$ and $21 \mathrm{~nm}$ into a thin (few $\mu \mathrm{m}$ thick) film of Poly(vinyl alcohol) (PVA) of $2.5 \mathrm{wt} \%$ mass concentration on a cover slide. This yields a threedimensional sparse distribution of isolated QDs with a mean lateral inter-particle distance which was significantly larger than the size of one dumbbell image. These QDs are typically five times brighter and by several orders of magnitudes more photo-stable than regular organic fluorescent dyes, and they exhibit intrinsic stochastic brightness fluctuations on time scales from nanoseconds to seconds which makes them ideal labels for SOFI.

We recorded movies of this sample with $30 \mathrm{~ms}$ frame time. In the sum image, we localized individual emitters and determined the orientation of their dumbbell pattern, and the distance between the dumbbell maxima as a function of orientation angle, see Fig. 4. This distance is orientation-dependent and can be approximated by a second order polynomial $d(\phi)=5.9+0.6(\phi-\pi / 2)^{2}$, where $d$ is given in units of pixel size $(160 \mathrm{~nm})$, and the orientation angle $\phi$ in radian. With a movie of 1700 frames,

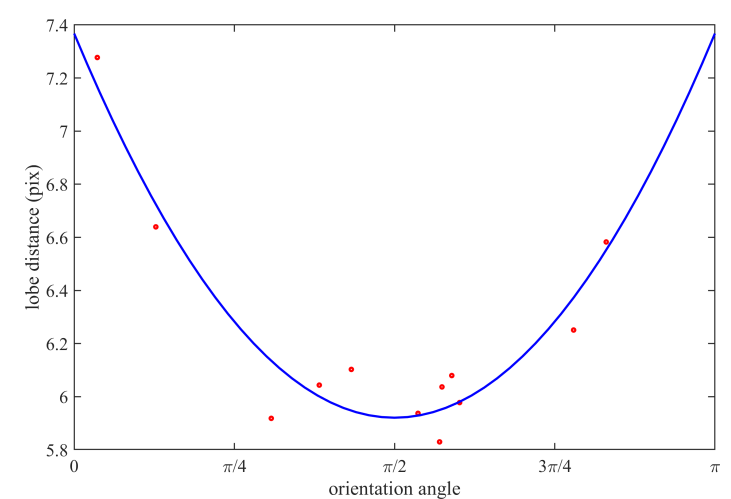

Fig. 4: Measured distances between dumbbell maxima as a function of orientation angle (red circles) for twelve quantum dots. Blue solid line shows fitted polynomial $5.9+0.6(\phi-\pi / 2)^{2}$. The scattering of the experimental points around the fitted curve reflect the limited fitting accuracy of the dumbbell fit (fitting a superposition of two symmetric Gaussian distributions) due to the finite number of collected photons.

we performed a spatio-temporal SOFI analysis as given by (5), using a discrete set of shift vectors with orientation angles $\phi$ between 0 and $165^{\circ}$ in steps of $15^{\circ}$ and shift distance $d(\phi)$. Before doing that, we generated from each raw image in the movie an interpolated image with ten times smaller pixel size by using a Fourier space upsampling as described in Ref. [19]. This allows for shifting images with respect to each other with 0.1 pixel resolution. The result for twelve individual QDs is shown in Fig. 5 where the top row shows the summed up wide-field images displaying the characteristic dumbbell pattern for each emitter, and the bottom row shows the stSOFI images, where the color indicates the orientation angle. The average size of one lobe in the dumbbell pattern, when fitted with a symmetric Gaussian distribution, is $0.224 \mu \mathrm{m}$. Due to the spatial cross-correlation, in stSOFI every particle appears as a single intensity peak with an average size that is $\sim 1.4$ times smaller than the size of a single lobe in the raw dumbbell image, as expected for second-order SOFI which predicts a PSF size reduction of $\sqrt{2}$.

Fig. 6 shows a comparison between the orientation angle as determined from fitting the orientation of the dumbbell pattern of an individual emitter, , and from determining the weighted average of the angle $\phi$ at the position of the peak intensity in the stSOFI image, using the twelve stSOFI intensities for the twelve discrete orientation angles as weights. As can be seen, there is fairly good agreement for for angles between $\approx 60^{\circ}$ and $\approx 120^{\circ}$ and a systematic non-linear deviation for smaller or larger angles. This is due to the increasing deformation of the pattern structure for angles far form the mid-point angle $\phi=90^{\circ}$, as can be especially seen in the first left image in the top row of Fig. 5. As can be seen, for large turn angels close to $0^{\circ}$ or $180^{\circ}$, the lobes of the dumbbell patterns develop coma-like deformations, so that when spatiotemporally correlating these patterns, the maximum cor- 


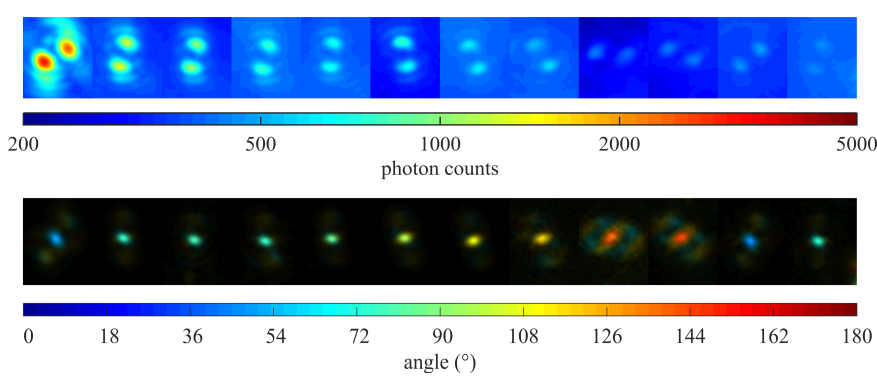

Fig. 5: Comparison between wide-field images (top row) and stSOFI images (bottom row) for twelve QDs. The color is calculated as weighted average using as weights the intensities of the twelve stSOFI images calculated for the twelve discrete orientation angles $\phi=0^{\circ}, 15^{\circ}, \ldots, 165^{\circ}$. Angles are counted counter-clockwise from the horizontal axis. Each sub-image has a width of $2.7 \mu \mathrm{m}$ and a height of $3.1 \mu \mathrm{m}$. The dynamic range $h$ of the used SPINDLE module is $3.18 \mu \mathrm{m}$, thus the shown angular range from $0^{\circ}$ to $180^{\circ}$ corresponds to this axial distance range of $3.18 \mu \mathrm{m}$.

relation will be achieved for a different angle than the angle obtained when fitting the dumbbell pattern directly with a superposition of two symmetric Gaussian intensity distributions. However, this is no principal restriction: For a given wavefront shaper, one has to fit the data in Fig. 5 with a sigmoidal curve and can then use it as calibration for recovering the correct angles (and thus axial distances) in stSOFI. However, the presented results clearly demonstrate the principal idea of stSOFI and its suitability for three-dimensional imaging.

Discussion and Conclusion. - In the current paper, we explained the general principle of stSOFI and focused on a proof of principle by taking data for single isolated quantum dots. We restricted ourselves to this kind of measurement on purpose, because it is the only way to directly reference the method against singleparticle localization along the optical axis by directly fitting the dumbbell orientation (as done in helical-wavefront 3D-STORM or PALM). Of course, for high-quality SOFI, one needs emitters that exhibit stochastic blinking on the time scale of the image acquisition time of the used microscope, and which have sufficient photo-stability for accumulating enough signal for a decent temporal correlation analysis. Although the QDs used in the current paper are in this respect optimal for SOFI, other emitters such as organic dyes $[23,24]$ or photo-switchable fluorescent proteins $[25,26]$ have been successfully used for SOFI in the past and should thus also be good candidates for stSOFI.

It remains the question what the optimal wave front shaper is for stSOFI. Ideally, one would like to have a PSF which consists of two identical copies of the same pattern, where each copy is as small as possible and the distance between copies is large with respect to the size of one copy. This will yield a maximum signal in the spatio-temporal correlation when the spatial correlation shift equals the center distance of the copies and the shift orientation is

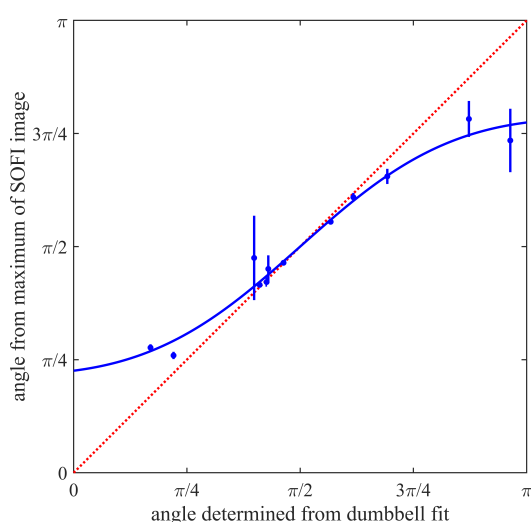

Fig. 6: Comparison between orientation angles as obtained by fitting the dumbbell pattern, and determined as a weighted average using the twelve stSOFI intensities for the twelve discrete orientation angles at stSOFI image peak position as weights. Error bars are determined by splitting the movie into ten batches of 170 frames and performing an stSOFI analysis on each batch separately. Different error widths are mainly caused by the different blinking behavior of the QDs. The solid line is a fit of a sigmoid, $\pi / 2+(\phi-\pi / 2) /\left[1+(\phi-\pi / 2)^{2} / \kappa^{2}\right]$, to the measurement. The fitted value of $\kappa$ is 2 .

equal to the corresponding relative orientation of the two copies (determined by the axial position of an emitter). In this sense, the helical wavefront shaper that produces two perfectly symmetric identical Gaussian intensity distributions seems to be the perfect choice for stSOFI. By minimizing the dynamic range $h$ one can increase the axial resolution, but for the prize of reducing the depth over which three-dimensional imaging is possible. This may be useful for thin samples, but problematic for thicker ones. In most cases, one will wish to adapt the dynamic range $h$ to the depth of focus of the used objective, thus sacrificing axial resolution but increasing the depth of threedimensional imaging.

The concept of stSOFI can be generalized also to other kinds of multi-modal imaging. As an example consider the case that, via wave-front shaping, the PSF of an emitter depends on its emission color [27]. For the sake of simplicity, let us assume that one wants to distinguish between a discrete number $N$ of colors, each color generating a distinct PSF $U_{\mu}(\boldsymbol{\rho})$, where the index $\mu=1, \ldots, N$ enumerates the color. Let us further assume that there exist a set of shift vectors $\mathbf{v}_{\mu}$ so that the orthogonality conditions $\left\langle U_{\mu}\left(\boldsymbol{\rho}+\mathbf{v}_{\nu}\right) U_{\mu}\left(\boldsymbol{\rho}-\mathbf{v}_{\nu}\right)\right\rangle=\delta_{\mu \nu} C_{\mu}$ are fulfilled, where angular brackets denote integration over $\boldsymbol{\rho}$, and $C_{\mu}$ is the overlap integral for two identical PSFs $U_{\mu}$ shifted by $\pm 2 \mathbf{v}_{\mu}$ to each other. It is obvious that, by using the discrete vectors $\mathbf{v}_{\mu}$ instead of the $\mathbf{v}(z)$ in eq. (5), the resulting $N$ stSOFI images will show only contributions from emitters with the correct color $\mu$ corresponding to the used shift vector $\mathbf{v}_{\mu}$. This concept can, of course, be applied to any emission/emitter characteristic which can be encoded into appropriate PSFs. Finally, we would like to mention that second-order stSOFI as expounded here can 
be straightforwardly generalized to higher-order stSOFI using higher-order temporal cumulants [10] (and thus increasing further spatial resolution), which will be done in a future publication.

\section{$* * *$}

JE is grateful to the Deutsche Forschungsgemeinschaft (DFG) for financial support via the Cluster of Excellence and DFG Research Center "Nanoscale Microscopy and Molecular Physiology of the Brain."

\section{REFERENCES}

[1] Hell S. W. and Wichmann J., Optics Letters, 19 (1994) 780 .

[2] Klar T. A. and Hell S. W., Optics Letters, 24 (1999) 954.

[3] Betzig E., Patterson G. H., Sougrat R., LindWasser O. W., Olenych S., Bonifacino J. S., Davidson M. W., Lippincott-Schwartz J. and Hess H. F., Science, 313 (2006) 1642.

[4] Hess S. T., Girirajan T. P. and Mason M. D., Biophysical Journal, 91 (2006) 4258.

[5] Rust M. J., Bates M. and Zhuang X., Nature Methods, 3 (2006) 793.

[6] Van De Linde S., Löschberger A., Klein T., HeidBreder M., Wolter S., Heilemann M. and Sauer M., Nature Protocols, 6 (2011) 991.

[7] Dertinger T., Colyer R., Iyer G., Weiss S. and EnDERLEin J., Proceedings of the National Academy of Sciences USA, 106 (2009) 22287.

[8] Willig K. I., Harke B., Medda R. and Hell S. W., Nature Methods, 4 (2007) 915.

[9] Wildanger D., Medda R., Kastrup L. and Hell S., Journal of Microscopy, 236 (2009) 35.

[10] Dertinger T., Xu J., Naini O. F., Vogel R. and WeIss S., Optical Nanoscopy, 1 (2012) 2.

[11] Dedecker P., Mo G. C. H., Dertinger T. and Zhang J., Proceedings of the National Academy of Sciences USA, 109 (2012) 10909.

[12] Huang B., Wang W., Bates M. and Zhuang X., Science, 319 (2008) 810.

[13] Juette M. F., Gould T. J., Lessard M. D., Mlodzianoski M. J., Nagpure B. S., Bennett B. T., Hess S. T. and Bewersdorf J., Nature Methods, 5 (2008) 527.

[14] Pavani S. R. P., Thompson M. A., Biteen J. S., Lord S. J., Liu N., Twieg R. J., Piestun R. and Moerner W., Proceedings of the National Academy of Sciences USA, 106 (2009) 2995.

[15] Shtengel G., Galbraith J. A., Galbraith C. G., Lippincott-Schwartz J., Gillette J. M., Manley S., Sougrat R., Waterman C. M., Kanchanawong P., Davidson M. W. et al., Proceedings of the National Academy of Sciences USA, 106 (2009) 3125.

[16] Dertinger T., Colyer R., Vogel R., Enderlein J. and Weiss S., Optics Express, 18 (2010) 18875.

[17] Duwé S., Vandenberg W. and Dedecker P., Chemical Communications, 53 (2017) 7242.
[18] Hebert B., Costantino S. and Wiseman P. W., Biophysical Journal, 88 (2005) 3601.

[19] Stein S. C., Huss A., Hähnel D., Gregor I. and ENDERLEIN J., Optics Express, 23 (2015) 16154.

[20] Geissbuehler S., Bocchio N. L., Dellagiacoma C., Berclaz C., Leutenegger M. and Lasser T., Optical Nanoscopy, 1 (2012) 4.

[21] Barsic A., Grover G. and Piestun R., Scientific Reports, 4 (2014) 5388.

[22] Pavani S. R. P. and Piestun R., Optics Express, 16 (2008) 22048.

[23] Dertinger T., Heilemann M., Vogel R., Sauer M. and Weiss S., Angewandte Chemie International Edition, 49 (2010) 9441.

[24] Dertinger T., Colyer R., Vogel R., Heilemann M., Sauer M., Enderlein J. and Weiss S., Superresolution optical fluctuation imaging (SOFI) in Nano-Biotechnology for Biomedical and Diagnostic Research (Springer) 2012 pp. 17-21.

[25] Moeyaert B. and Dedecker P., pcSOFI as a smart label-based superresolution microscopy techique in Photoswitching Proteins (Springer) 2014 pp. 261-276.

[26] Hertel F., Mo G. C., Duwé S., Dedecker P. and Zhang J., Cell Reports, 14 (2016) 390.

[27] Shechtman Y., Weiss L. E., Backer A. S., Lee M. Y. and Moerner W., Nature Photonics, 10 (2016) 590. 